\title{
Pseudo-Entropy in Free Quantum Field Theories
}

\author{
Ali Mollabashi®,${ }^{1,2}$ Noburo Shiba, ${ }^{2}$ Tadashi Takayanagi $\odot,{ }^{2,3,4}$ Kotaro Tamaoka $\odot,{ }^{2}$ and Zixia Wei ${ }^{2}$ \\ ${ }^{1}$ Max-Planck-Institut for Physics, Werner-Heisenberg-Institut, 80805 Munich, Germany \\ ${ }^{2}$ Yukawa Institute for Theoretical Physics, Kyoto University, Kitashirakawa Oiwakecho, Sakyo-ku, Kyoto 606-8502, Japan \\ ${ }^{3}$ Inamori Research Institute for Science, 620 Suiginya-cho, Shimogyo-ku, Kyoto 600-8411, Japan \\ ${ }^{4}$ Kavli Institute for the Physics and Mathematics of the Universe, University of Tokyo, Kashiwa, Chiba 277-8582, Japan
}

(Received 25 November 2020; accepted 8 January 2021; published 26 February 2021)

\begin{abstract}
Pseudo-entropy is an interesting quantity with a simple gravity dual, which generalizes entanglement entropy such that it depends on both an initial and a final state. Here we reveal the basic properties of pseudo-entropy in quantum field theories by numerically calculating this quantity for a set of twodimensional free-scalar field theories and the Ising spin chain. We extend the Gaussian method for pseudoentropy in free-scalar theories with two parameters: mass $m$ and dynamical exponent $z$. This computation finds two novel properties of pseudo-entropy which we conjecture to be universal in field theories, in addition to an area law behavior. One is a saturation behavior and the other one is nonpositivity of the difference between pseudo-entropy and averaged entanglement entropy. Moreover, our numerical results for the Ising chain imply that pseudo-entropy can play a role as a new quantum order parameter which detects whether two states are in the same quantum phase or not.
\end{abstract}

DOI: 10.1103/PhysRevLett.126.081601

Introduction.-Entanglement entropy in quantum many-body systems plays significant roles in various subjects of theoretical physics, such as condensed matter physics [1-3], particle physics [4-8], and gravitational physics [9-12]. In the anti-de Sitter/conformal field theory (AdS/CFT) correspondence, [13], entanglement entropy is equal to the area of a minimal surface $[14,15]$. This directly relates geometric structures in quantum many-body systems to those of spacetimes in gravitational theories.

Recently, a new geometric connection between a minimal area surface and a novel quantity, called pseudo-entropy, has been found via AdS/CFT [16]. The pseudo-entropy is a generalization of entanglement entropy to a transition between the initial state $\left|\psi_{1}\right\rangle$ and the final state $\left|\psi_{2}\right\rangle$. First we introduce the transition matrix $\tau^{1 \mid 2}$ :

$$
\tau^{1 \mid 2}=\frac{\left|\psi_{1}\right\rangle\left\langle\psi_{2}\right|}{\left\langle\psi_{2} \mid \psi_{1}\right\rangle} .
$$

We divide the total Hilbert space $\mathcal{H}_{\text {tot }}$ into two parts $A$ and $B$ as we do so to define entanglement entropy; i.e., $\mathcal{H}_{\text {tot }}=\mathcal{H}_{A} \otimes \mathcal{H}_{B}$. We introduce the reduced transition

Published by the American Physical Society under the terms of the Creative Commons Attribution 4.0 International license. Further distribution of this work must maintain attribution to the author(s) and the published article's title, journal citation, and DOI. Funded by SCOAP ${ }^{3}$. matrix $\tau_{A}^{1 \mid 2}=\operatorname{Tr}_{B}\left[\tau^{1 \mid 2}\right]$ by tracing out $\mathcal{H}_{B}$. Finally, pseudo-entropy is defined by

$$
S\left(\tau_{A}^{1 \mid 2}\right)=-\operatorname{Tr}\left[\tau_{A}^{1 \mid 2} \log \tau_{A}^{1 \mid 2}\right] .
$$

Note that when $\left|\psi_{1}\right\rangle=\left|\psi_{2}\right\rangle$, this quantity is equal to the ordinary entanglement entropy. Even though this expression (2) looks like the von Neumann entropy, this takes complex values in general because $\tau_{A}^{1 \mid 2}$ is no longer Hermitian. However, when we construct the initial and final state by a Euclidean path integral with a real valued action, $S\left(\tau_{A}^{1 \mid 2}\right)$ turns out to be positive [16], which is the case we will focus on in this Letter. Moreover, it was found that the pseudoentropy for holographic CFTs can be computed as the areas of minimal surfaces in time-dependent Euclidean asymptotically anti-de Sitter backgrounds [16]. Such a timedependent Euclidean space is dual to an inner product $\left\langle\psi_{2} \mid \psi_{1}\right\rangle$ via AdS/CFT [13]. In addition to the above importance in gravity, pseudo-entropy has an intriguing interpretation from the quantum information viewpoint, as a measure of quantum entanglement for intermediate states between the initial and the final state [16]. In this Letter we would like to pursue the next obviously important task, namely, to uncover basic properties of pseudo-entropy in quantum many-body systems, including quantum field theories and condensed matter systems. For technical details used in this Letter, refer to [17]

Free-scalar field theory.-Consider free-scalar field theory in two dimensions as our first example. We take into account two parameters in the free-scalar theory, which are the mass $m$ and the dynamical exponent $z$. At $z=1$, 
this describes the relativistic scalar field, while for $z>1$, it is called Lifshitz scalar field, which is invariant under the Lifshitz scaling symmetry $t \rightarrow \lambda^{z} t, x \rightarrow \lambda x$ in the $m \rightarrow 0$ limit. Its Hamiltonian is written as

$$
H=\frac{1}{2} \int d x\left[\pi^{2}+\left(\partial_{x}^{z} \phi\right)^{2}+m^{2 z} \phi^{2}\right],
$$

where $\phi$ and $\pi$ are the scalar field and its momentum.

In order to do concrete calculations, we consider its lattice regularization [18-21] given by the Hamiltonian:

$H=\sum_{i=1}^{N}\left\{\frac{\pi_{i}^{2}}{2}+\frac{m^{2 z}}{2} \phi_{n}^{2}+\frac{1}{2}\left[\sum_{k=0}^{z}(-1)^{z+k}\left(\begin{array}{l}z \\ k\end{array}\right) \phi_{i-1+k}\right]^{2}\right\}$

where $N$ is the total lattice size. We define $N_{A}$ to be the lattice size of subsystem $A$. These models are straightforwardly generalized to higher dimensions $[18,20]$.

It is known that we can calculate the entanglement entropy in free-field theories from correlation functions on $A$ when a quantum state is described by a Gaussian wave functional [22]. Even though for pseudo-entropy we consider a transition matrix instead of a density matrix, we can remarkably extend this Gaussian calculation via an analytic continuation. This makes numerical computations of pseudo-entropy possible, playing a major role below.

Two point functions of $\phi$ and $\pi$ compose the $2 N_{A} \times 2 N_{A}$ matrix $\Gamma$ :

$$
\Gamma=\left(\begin{array}{cc}
X & R \\
R^{T} & P
\end{array}\right)
$$

where

$$
\begin{aligned}
X_{i j} & =\operatorname{Tr}\left[\phi_{i} \phi_{j} \tau_{A}^{1 \mid 2}\right], \quad P_{i j}=\operatorname{Tr}\left[\pi_{i} \pi_{j} \tau_{A}^{1 \mid 2}\right], \\
R_{i j} & =\frac{1}{2} \operatorname{Tr}\left[\left(\phi_{i} \pi_{j}+\pi_{i} \phi_{j}\right) \tau_{A}^{1 \mid 2}\right] .
\end{aligned}
$$

As opposed to the standard case where $\tau_{A}^{1 / 2}$ is given by a Hermitian density matrix $\rho_{A}$, we find that the matrix $R$ takes complex values, though $X$ and $P$ are real symmetric matrices. Therefore, we consider a complexified symplectic transformation $\operatorname{Sp}\left(2 N_{A}, \mathbb{C}\right)$ to diagonalize $\Gamma$ into the form

$$
\Gamma \rightarrow\left(\begin{array}{ll}
\nu & 0 \\
0 & \nu
\end{array}\right)
$$

where $\nu$ is a diagonal matrix and we write its diagonal components as $\nu_{i}=\frac{1}{2} \operatorname{coth}\left(\epsilon_{i} / 2\right)$ [23]. Practically, we can obtain $\nu_{i}$ from the fact that the eigenvalues of the following rearranged matrix are $\pm \nu_{i}$ :

$$
\left(\begin{array}{cc}
i R^{T} & i P \\
-i X & -i R
\end{array}\right)
$$

In the examples which we focus on below, $\nu_{i}$ and $\epsilon_{i}$ always take positive real values. Finally, the pseudoentropy is computed by the formula

$$
S\left(\tau_{A}^{1 \mid 2}\right)=\sum_{i=1}^{N_{A}}\left[\frac{\epsilon_{i}}{e^{\epsilon_{i}}-1}-\log \left(1-e^{-\epsilon_{i}}\right)\right]=\sum_{i=1}^{N_{A}}\left[\left(\nu_{i}+\frac{1}{2}\right) \log \left(\nu_{i}+\frac{1}{2}\right)-\left(\nu_{i}-\frac{1}{2}\right) \log \left(\nu_{i}-\frac{1}{2}\right)\right] .
$$

This Gaussian calculation of pseudo-entropy can also be justified by a more direct approach, the operator method $[25,26]$. Though it has not been proven rigorously that performing the analytic continuation used in this Gaussian calculation is possible, we can directly derive the same formula by the operator method without using the analytic continuation. In our analysis, we take $\left|\psi_{1}\right\rangle$ and $\left|\psi_{2}\right\rangle$ to be ground states for various values of the mass $m$ and dynamical exponent $z$, which we denote by $\left(m_{1}, z_{1}\right)$ and $\left(m_{2}, z_{2}\right)$.

Let us first start with the relativistic setups $z_{1}=z_{2}=1$ and $m_{1} \neq m_{2}$. We take the total system to be a circle length $L$ and define a subsystem $A$ to be a length $l$ interval on this circle. We write the UV cutoff (lattice spacing) as $\epsilon$ such that $L=N \epsilon$ and $l=N_{A} \epsilon$. Our numerical analysis reveals the general behavior of pseudo-entropy.

$$
S\left(\tau_{A}^{1 \mid 2}\right)=\frac{1}{3} \log \left[\frac{L}{\pi \epsilon} \sin \left(\frac{\pi l}{L}\right)\right]+f\left(m_{1}, m_{2}, L, l\right),
$$

where the first term on the right-hand side coincides with the known behavior of entanglement entropy in twodimensional CFT with the central charge $c=1[3,6]$, while the second term is a constant term which depends on the relevant parameters. For confirmation of this behavior, refer to Fig. 1, where the first logarithmic term in Eq. (8) gives a

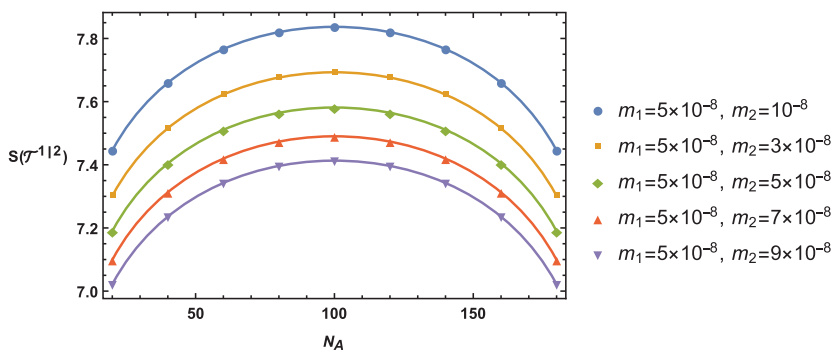

FIG. 1. $S\left(\tau_{A}^{1 \mid 2}\right)$ as a function of the size of the subsytem $N_{A}$. We set $N=200$ and $z_{1}=z_{2}=1$. The curves are $c_{1} \ln \left[(N / \pi) \sin \left(\pi N_{A} / N\right)\right]+c_{0}$, where $c_{1} \simeq 0.3333$ and $6.028<$ $c_{0}<6.453$. 
dominant $l$ dependence for small masses. This shows that the leading logarithmic divergence, which is equivalent to the area law, is robust for the pseudo-entropy. For small values of masses, our numerical calculations determine analytical structures of the function $f\left(m_{1}, m_{2}, L, l\right)$. When we consider the almost massless limit $m_{1,2} L \ll 1$, we have

$$
f\left(m_{1}, m_{2}, L, l\right) \simeq-\frac{1}{2} \log \left[\frac{m_{1}+m_{2}}{2} L\right] .
$$

This logarithmic behavior is due to the zero mode of scalar field and the above formula agrees with the known result of entanglement entropy in Ref. [27]. When the mass is small such that $m_{1,2} L \sim 1$ and $m_{1,2} l \ll 1$, we can semianalytically [28] find the $l$ dependence,

$$
\begin{aligned}
& f\left(m_{1}, m_{2}, L, l\right) \\
& \simeq \frac{1}{2} \log \left[-\frac{m_{1}^{2} \log \left[m_{1} l\right]-m_{2}^{2} \log \left[m_{2} l\right]}{m_{1}^{2}-m_{2}^{2}}\right]+f_{0}\left(m_{1}, m_{2}, L\right),
\end{aligned}
$$

where the final term $f_{0}$ does not depend on $l$. This expression again reproduces the known $\frac{1}{2} \log [-\log (m l)]$ term [29] in the entanglement entropy.

Now we turn to the dynamical exponent $\left(z_{1}, z_{2}\right)$ to describe the Lifshitz scalar theory. When $z_{1}=z_{2}$, the pseudo-entropy gets larger as the dynamical exponent increases, as in the upper graph of Fig. 2. When we fix $z_{1}$ and increase $z_{2}$, the pseudo-entropy approaches a certain finite value, as can be seen from the lower graph in Fig. 2. We call this phenomenon saturation. The saturation occurs when we fix $\left|\psi_{1}\right\rangle$ and consider a limit where the entanglement of $\left|\psi_{2}\right\rangle$ gets larger. The two graphs in Fig. 3 demonstrate the saturations when we take different two
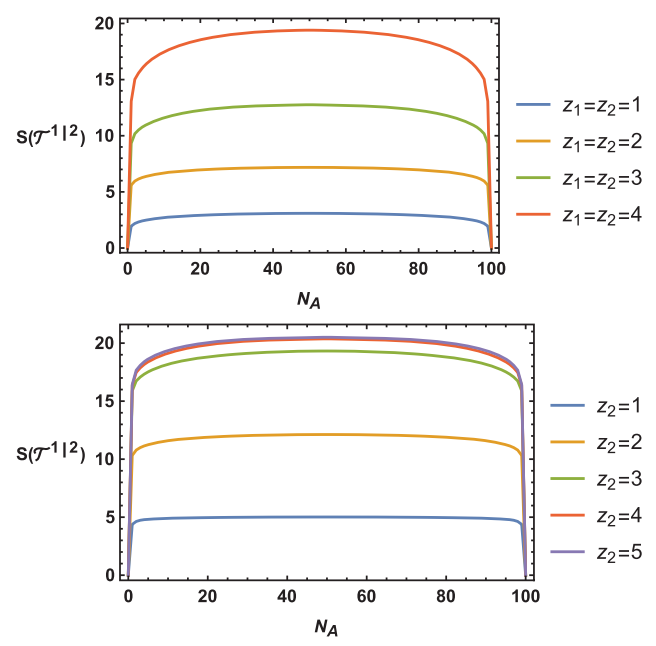

FIG. 2. The upper plot shows the pseudo-entropy as a function of the subsystem size $N_{A}$ when we chose $m_{1}=10^{-3}$ and $m_{2}=10^{-5}$ for various values of $z_{1}=z_{2}$. The lower plot shows the pseudo-entropy when we set $z_{1}=3$ and $m_{1}=m_{2}=10^{-5}$. We chose the total system $N=100$. limits of $m_{2} \rightarrow 0$ and $z_{2} \rightarrow \infty$, respectively. This saturation in our free-scalar field theory implies that the behavior of pseudo-entropy qualitatively looks like

$$
S\left(\tau_{A}^{1 \mid 2}\right) \sim \min \left[S\left(\rho_{A}^{1}\right), S\left(\rho_{A}^{2}\right)\right] .
$$

From our numerical results, we can find one more basic property of pseudo-entropy by introducing the difference:

$$
\Delta S_{12} \equiv S\left(\tau_{A}^{1 \mid 2}\right)-\frac{S\left(\rho_{A}^{1}\right)+S\left(\rho_{A}^{2}\right)}{2} .
$$

If $\left|\psi_{1}\right\rangle$ and $\left|\psi_{2}\right\rangle$ are very close to a state $\left|\psi_{0}\right\rangle$, such that $\delta \tau_{A}=\tau_{A}^{1 / 2}-\rho_{A}^{0}$ is very small, then we can derive a first law like relation:

$$
S\left(\tau_{A}^{1 \mid 2}\right)-S\left(\rho_{A}^{0}\right) \simeq \frac{\left\langle\psi_{2}\left|H_{A}\right| \psi_{1}\right\rangle}{\left\langle\psi_{2} \mid \psi_{1}\right\rangle}+O\left(\left(\delta \tau_{A}\right)^{2}\right)
$$

as in the first law of entanglement entropy [30-32]. Here we introduced the modular Hamiltonian $H_{A}=$ $-\log \rho_{A}^{0}-S\left(\rho_{A}^{0}\right)$. The linear combination (11) is special such that it cancels out in this linear difference (12), leaving only the quadratic order as $\Delta S_{12}=O\left(\left(\delta \tau_{A}\right)^{2}\right)$.

In general, this quadratic difference $\Delta S_{12}$ is not guaranteed to be positive definite. Indeed, we can confirm that both signs are possible even in a 2-qubit example. However, in all of our numerical results in the free-scalar field theory (3), we observe its nonpositivity $\Delta S_{12} \leq 0$ when we vary the masses and dynamical exponents, as depicted in Fig. 4. Also, in the small mass limit (9), this nonpositivity is satisfied.
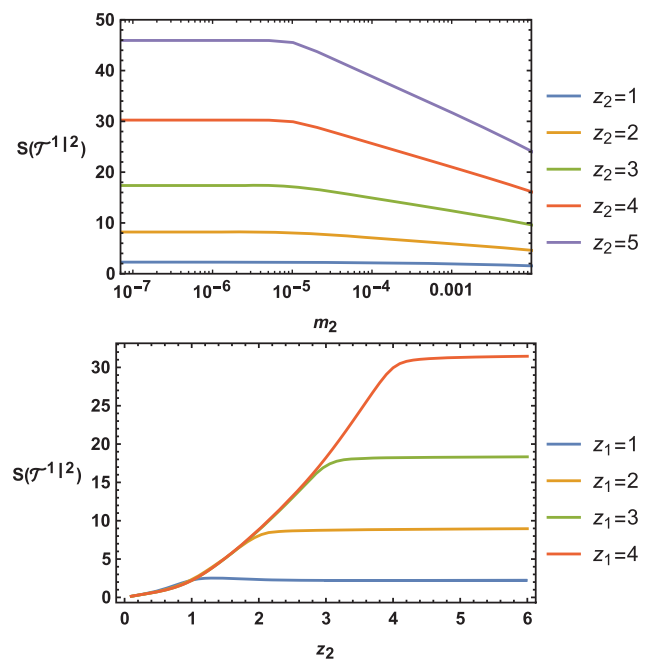

FIG. 3. The upper graph shows the pseudo-entropy as a function of $m_{2}$ when we set $z_{1}=1$ and $m_{1}=10^{-5}$. The lower graph depicts the pseudo-entropy as a function of $z_{2}$ when we set $m_{1}=m_{2}=10^{-5}$. We chose $N_{A}=50$ and $N=\infty$. 



FIG. 4. The plots of the difference $\Delta S_{12}$ as a function of $m_{2}-$ $m_{1}$ (upper) and $z_{2}$ (lower). We set $m_{1}=10^{-5}$ and $z_{1}=z_{2}$ in the upper graph. We chose $m_{1}=m_{2}=10^{-5}$ in the lower graph.

Pseudo-entropy in perturbed CFT.-To investigate the behavior of pseudo-entropy more, consider a perturbation in a two-dimensional CFT. We assume that the subsystem $A$ is a length $l$ interval and the CFT is defined on $R^{2}$. The perturbation is expressed as $\lambda \int d t d x O(t, x)$, where $O$ is a primary operator and $\lambda$ is a small perturbation parameter. We choose $\left|\psi_{1}\right\rangle$ as the original CFT vacuum and $\left|\psi_{2}\right\rangle$ is the new vacuum obtained by this perturbation. Since one point function vanishes in a CFT, there is no $O(\lambda)$ term in the differences $S\left(\tau_{A}^{1 \mid 2}\right)-S\left(\rho_{A}^{1}\right)$. Moreover, at the order $O\left(\lambda^{2}\right)$, we can show $S\left(\tau_{A}^{1 \mid 2}\right)-S\left(\rho_{A}^{1}\right) \leq 0$. This result is universal because it only involves two point functions in a CFT.

In particular, if we consider an exactly marginal perturbation, we find that the coefficient of the logarithmically divergent terms is changed:

$$
S\left(\tau_{A}^{1 \mid 2}\right)=\frac{c}{3} f(\lambda) \log \frac{l}{\epsilon}+\text { const },
$$

The conformal perturbation shows $f(\lambda)=1+g \lambda^{2}+$ $O\left(\lambda^{3}\right)$ with $g<0$ in the $\lambda \rightarrow 0$ limit. We can also derive the same behavior from the holographic calculation of pseudo-entropy in Janus solutions [33-38]. In this way, we can confirm $\Delta S_{12} \leq 0$ for exactly marginal perturbations.

Pseudo-entropy in Ising model.-As another class of basic quantum many-body systems, we would like to consider a transverse Ising spin chain model. In the continuum limit near the critical point, this model is known to be equivalent to the two-dimensional free-fermion CFT [39]. Its Hamiltonian can be written as

$$
H=-J \sum_{i=0}^{N-1} \sigma_{i}^{z} \sigma_{i+1}^{z}-h \sum_{i=0}^{N-1} \sigma_{i}^{x},
$$
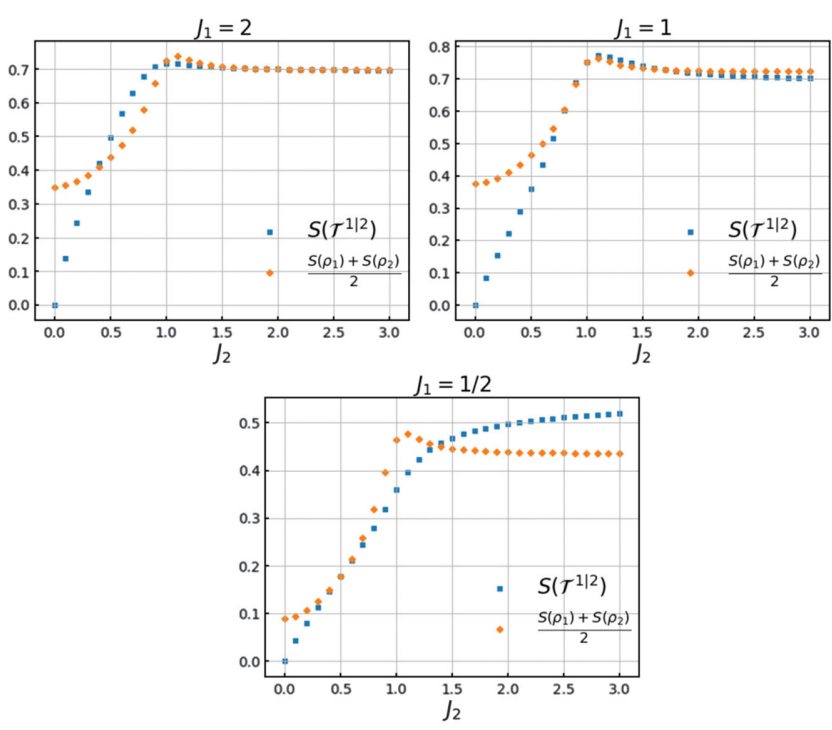

FIG. 5. Pseudo-entropy and average of entanglement for a single interval. Here, we choose $N=16, N_{A}=8, h_{1}=h_{2}=1$. We set $J_{1}=2$ (upper left), $J_{1}=1$ (upper right), and $J_{1}=1 / 2$ (lower). The horizontal axis is the value of $J_{2}$. The blue dots show the pseudo-entropy $S\left(\mathcal{T}_{A}^{1 \mid 2}\right)$ and the orange dots show the average of the entanglement entropy $\left[S\left(\rho_{A}^{1}\right)+S\left(\rho_{A}^{2}\right)\right] / 2$.

where the spins are be labeled by $i=0,1,2, \ldots, N-1$ and the $\sigma_{i}^{z}$ is Pauli operator on $i$ with eigenvalues \pm 1 . We impose the periodic boundary condition. Note that the quantum critical point is situated at $J=h$ in the continuum limit, where $J>h$ is the ferromagnetic phase, while $J<h$ describes the paramagnetic phase.

We calculate the pseudo-entropy $S\left(\tau_{A}^{1 \mid 2}\right)$ by choosing $\left|\psi_{1}\right\rangle$ and $\left|\psi_{2}\right\rangle$ to be the ground states for $(J, h)=\left(J_{1}, h_{1}\right)$ and $\left(J_{2}, h_{2}\right)$, respectively. The subsystem $A$ is assumed to be a single interval with $N_{A}$ spins. We show numerical results in Fig. 5 (we used the PYTHON package QUSPIN [40] in our computation).

From the numerical results, we can observe the saturation $S\left(\tau_{A}^{1 \mid 2}\right) \simeq \log 2$ in the $J_{2} \rightarrow \infty$ limit when $J_{1}>1$. Moreover, we can confirm that the difference (11) satisfies $\Delta S_{12} \leq 0$ when $\left(J_{1}, h_{1}\right)$ and $\left(J_{2}, h_{2}\right)$ are in the same phase, i.e., $\left(J_{1}-h_{1}\right)\left(J_{2}-h_{2}\right)>0$. However, we can have $\Delta S_{12}>0$ when they belong to two different phases, i.e., $\left(J_{1}-h_{1}\right)\left(J_{2}-h_{2}\right)<0$. This implies that the sign of the difference $\Delta S_{12}$ can provide an order parameter which tells us whether the two states $\left|\psi_{1}\right\rangle$ and $\left|\psi_{2}\right\rangle$ are in the same phase or not. This result is also expected to hold when considering two ground states of 2D free Majorana fermion theories with different mass as long as they belong to the same phase [41], since free Majorana fermion can be obtained as a scaling limit of transverse Ising chain after Jordan-Wigner transformations.

Discussions. - In this Letter we have uncovered basic properties of pseudo-entropy in quantum field theories by focusing on numerical calculations in a class of free-scalar field theories and the Ising spin chain. We would like to 
conjecture that the properties, area law, saturation, and nonpositivity of $\Delta S_{12}$, which we found for free-scalar field theories, will be universal also for any quantum field theory. It will be an important future problem to study pseudo-entropy in a broader class of field theories and test the above properties. Moreover, our results for Ising spin chain imply that we can classify different phases in quantum many-body systems from the calculations of pseudo-entropy. This originates from our expectation that the pseudo-entropy helps us to probe the difference of structures of quantum entanglement between two states. One obvious future direction will be to analyze the pseudoentropy in topological phases, to see if it can play a role of topological order parameter.

We are grateful to Seishiro Ono, Hong Yang, and Chi Zhang for useful discussions and to Yoshifumi Nakata, Tatsuma Nishioka, and Yusuke Taki for communications on this article. T. T. is supported by Grant-in-Aid for JSPS Fellows No. 19F19813. K. T. and T. T. are supported by the Simons Foundation through the "It from Qubit" collaboration. T. T. is supported by Inamori Research Institute for Science and World Premier International Research Center Initiative (WPI Initiative) from the Japan Ministry of Education, Culture, Sports, Science and Technology (MEXT). N.S. and T. T. are supported by JSPS Grant-in-Aid for Scientific Research (A) No. 16H02182. A. M. and T. T. are also supported by JSPS Grant-in-Aid for Challenging Research (Exploratory) 18K18766. K. T. is also supported by JSPS Grant-in-Aid for Research Activity start-up 19K23441. A. M. is generously supported by Alexander von Humboldt foundation via a postdoctoral fellowship. N. S. is also supported by JSPS KAKENHI Grant No. JP19K14721. Z.W. is supported by the ANRI Fellowship and Grant-in-Aid for JSPS Fellows No. 20J23116.

[1] G. Vidal, J. I. Latorre, E. Rico, and A. Kitaev, Entanglement in Quantum Critical Phenomena, Phys. Rev. Lett. 90, 227902 (2003).

[2] A. Kitaev and J. Preskill, Topological Entanglement Entropy, Phys. Rev. Lett. 96, 110404 (2006); M. Levin and X. G. Wen, Detecting Topological Order in a Ground State Wave Function, Phys. Rev. Lett. 96, 110405 (2006).

[3] P. Calabrese and J. L. Cardy, Entanglement entropy and quantum field theory, J. Stat. Mech. (2004) P06002.

[4] L. Bombelli, R. K. Koul, J. Lee, and R. D. Sorkin, A quantum source of entropy for black holes, Phys. Rev. D 34, 373 (1986).

[5] M. Srednicki, Entropy and Area, Phys. Rev. Lett. 71, 666 (1993).

[6] C. Holzhey, F. Larsen, and F. Wilczek, Geometric and renormalized entropy in conformal field theory, Nucl. Phys. B424, 443 (1994).

[7] H. Casini and M. Huerta, Entanglement entropy in free quantum field theory, J. Phys. A 42, 504007 (2009).
[8] T. Nishioka, Entanglement entropy: Holography and renormalization group, Rev. Mod. Phys. 90, 035007 (2018).

[9] T. Nishioka, S. Ryu, and T. Takayanagi, Holographic entanglement entropy: An overview, J. Phys. A 42, 504008 (2009); M. Rangamani and T. Takayanagi, Holographic entanglement entropy, Lect. Notes Phys. 931, 1 (2017).

[10] M. Van Raamsdonk, Lectures on gravity and entanglement, arXiv:1609.00026.

[11] D. Harlow, TASI lectures on the emergence of bulk physics in AdS/CFT, Proc. Sci. TASI2017 (2018) 002.

[12] A. Almheiri, T. Hartman, J. Maldacena, E. Shaghoulian, and A. Tajdini, The entropy of Hawking radiation, arXiv: 2006.06872.

[13] J. M. Maldacena, The large $N$ limit of superconformal field theories and supergravity, Int. J. Theor. Phys. 38, 1113 (1999); Adv. Theor. Math. Phys. 2, 231 (1998).

[14] S. Ryu and T. Takayanagi, Holographic Derivation of Entanglement Entropy from AdS/CFT, Phys. Rev. Lett. 96, 181602 (2006); Aspects of holographic entanglement entropy, J. High Energy Phys. 08 (2006) 045.

[15] V. E. Hubeny, M. Rangamani, and T. Takayanagi, A covariant holographic entanglement entropy proposal, J. High Energy Phys. 07 (2007) 062.

[16] Y. Nakata, T. Takayanagi, Y. Taki, K. Tamaoka, and Z. Wei, New holographic generalization of entanglement entropy, Phys.Rev.D 103, 026005 (2021).

[17] See Supplemental Material at http://link.aps.org/supplemental/ 10.1103/PhysRevLett.126.081601 for technical details used in this Letter.

[18] M. R. Mohammadi Mozaffar and A. Mollabashi, Entanglement in Lifshitz-type quantum field theories, J. High Energy Phys. 07 (2017) 120.

[19] T. He, J. M. Magan, and S. Vandoren, Entanglement entropy in Lifshitz theories, SciPost Phys. 3, 034 (2017).

[20] M. R. Mohammadi Mozaffar and A. Mollabashi, Logarithmic negativity in Lifshitz harmonic models, J. Stat. Mech. (2018) 053113.

[21] M. R. Mohammadi Mozaffar and A. Mollabashi, Entanglement evolution in Lifshitz-type scalar theories, J. High Energy Phys. 01 (2019) 137.

[22] K. Audenaert, J. Eisert, M. Plenio, and R. Werner, Entanglement properties of the harmonic chain, Phys. Rev. A 66, 042327 (2002); A. Botero and B. Reznik, Spatial structures and localization of vacuum entanglement in the linear harmonic chain, Phys. Rev. A 70, 052329 (2004); M. B. Plenio, J. Eisert, J. Dreissig, and M. Cramer, Entropy, Entanglement, and Area: Analytical Results for Harmonic Lattice Systems, Phys. Rev. Lett. 94, 060503 (2005); I. Peschel and V. Eisler, Reduced density matrices and entanglement entropy in free lattice models, J. Phys. A 42, 504003 (2009).

[23] A similar method to work out the entanglement spectrum for Gaussian states is based on Williamson's theorem [24] where a symplectic transformation diagonalizes the correlation matrix $\Gamma$.

[24] J. Williamson, On the algebraic problem concerning the normal forms of linear dynamical systems, Am. J. Math. 58, 141 (1936). 
[25] N. Shiba, Entanglement entropy of disjoint regions in excited states: An operator method, J. High Energy Phys. 12 (2014) 152.

[26] N. Shiba, Direct calculation of mutual information of distant regions, J. High Energy Phys. 09 (2020) 182.

[27] H. Casini and M. Huerta, A finite entanglement entropy and the c-theorem, Phys. Lett. B 600, 142 (2004).

[28] S. Chapman, J. Eisert, L. Hackl, M. P. Heller, R. Jefferson, H. Marrochio, and R. C. Myers, Complexity and entanglement for thermofield double states, SciPost Phys. 6, 034 (2019); M. R. Mohammadi Mozaffar and A. Mollabashi, On time scaling of entanglement entropy in Lifshitz theories (to be published).

[29] H. Casini and M. Huerta, Entanglement and alpha entropies for a massive scalar field in two dimensions, J. Stat. Mech. (2005) P12012.

[30] J. Bhattacharya, M. Nozaki, T. Takayanagi, and T. Ugajin, Thermodynamical Property of Entanglement Entropy for Excited States, Phys. Rev. Lett. 110, 091602 (2013).

[31] D. D. Blanco, H. Casini, L. Y. Hung, and R. C. Myers, Relative entropy and holography, J. High Energy Phys. 08 (2013) 060.

[32] G. Wong, I. Klich, L. A. Pando Zayas, and D. Vaman, Entanglement temperature and entanglement entropy of excited states, J. High Energy Phys. 12 (2013) 020.
[33] D. Z. Freedman, C. Nunez, M. Schnabl, and K. Skenderis, Fake supergravity and domain wall stability, Phys. Rev. D 69, 104027 (2004).

[34] D. Bak, M. Gutperle, and S. Hirano, A dilatonic deformation of $\operatorname{AdS}(5)$ and its field theory dual, J. High Energy Phys. 05 (2003) 072.

[35] D. Bak, M. Gutperle, and S. Hirano, Three dimensional Janus and time-dependent black holes, J. High Energy Phys. 02 (2007) 068.

[36] A. B. Clark, D. Z. Freedman, A. Karch, and M. Schnabl, Dual of the Janus solution: An interface conformal field theory, Phys. Rev. D 71, 066003 (2005).

[37] A. Clark and A. Karch, Super Janus, J. High Energy Phys. 10 (2005) 094.

[38] E. D'Hoker, J. Estes, and M. Gutperle, Ten-dimensional supersymmetric Janus solutions, Nucl. Phys. B757, 79 (2006).

[39] S. Sachdev, Quantum Phase Transitions (Cambridge University Press, Cambridge, England, 2001).

[40] P. Weinberg and M. Bukov, QuSpin: A Python package for dynamics and exact diagonalisation of quantum many body systems part I: Spin chains, SciPost Phys. 2, 003 (2017).

[41] A. Mollabashi, N. Shiba, T. Takayanagi, K. Tamaoka, and Z. Wei (to be published). 